\title{
Low-temperature crystal and magnetic structures of the magnetoelectric material $\mathrm{Fe}_{4} \mathrm{Nb}_{2} \mathrm{O}_{9}$
}

\author{
Rajesh Jana, ${ }^{1}$ Denis Sheptyakov, ${ }^{2}$ Xiaoyan Ma, ${ }^{1}$ Jose A. Alonso,,${ }^{3, *}$ Maocai Pi, ${ }^{4}$ Angel Muñoz, ${ }^{5}$ Ziyi Liu, ${ }^{1}$ \\ Linlin Zhao, ${ }^{1,6} \mathrm{Na} \mathrm{Su},{ }^{1,6}$ Shifeng Jin, ${ }^{1,6}$ Xiaobai Ma, ${ }^{7}$ Kai Sun, ${ }^{7}$ Dongfeng Chen, ${ }^{7}$ Shuai Dong, ${ }^{8}$ \\ Yisheng Chai, ${ }^{4, \dagger}$ Shiliang $\mathrm{Li},{ }^{1,6,9}$ and Jinguang Cheng $\circledast^{1,6,9, \$}$ \\ ${ }^{1}$ Beijing National Laboratory for Condensed Matter Physics and Institute of Physics, Chinese Academy of Sciences, Beijing 100190, China \\ ${ }^{2}$ Laboratory for Neutron Scattering and Imaging, Paul Scherrer Institute, 5232 Villigen PSI, Switzerland \\ ${ }^{3}$ Instituto de Ciencia de Materiales de Madrid, CSIC, Cantoblanco, E-28049 Madrid, Spain \\ ${ }^{4}$ Low Temperature Physics Laboratory, College of Physics, and Center of Quantum Materials and Devices, \\ Chongqing University, Chongqing 401331, China \\ ${ }^{5}$ Universidad Carlos III, Avenida Universidad 30, E-28911, Leganés-Madrid, Spain \\ ${ }^{6}$ School of Physical Sciences, University of Chinese Academy of Sciences, Beijing 100190, China \\ ${ }^{7}$ Department of Nuclear Physics, China Institute of Atomic Energy, Beijing 102413, China \\ ${ }^{8}$ School of Physics, Southeast University, Nanjing 211189, China \\ ${ }^{9}$ Songshan Lake Materials Laboratory, Dongguan, Guangdong 523808, China
}

(Received 26 April 2019; revised manuscript received 24 July 2019; published 26 September 2019)

\begin{abstract}
$\mathrm{Fe}_{4} \mathrm{Nb}_{2} \mathrm{O}_{9}$ was recently reported to be a new magnetoelectric material with two distinct dielectric anomalies located at $T_{N} \approx 90 \mathrm{~K}$ for an antiferromagnetic transition and $T_{\text {str }} \approx 77 \mathrm{~K}$ of unknown origin, respectively. By analyzing low-temperature neutron-powder-diffraction data, here we determined its magnetic structure below $T_{N}$ and uncovered the origin of the second dielectric anomaly as a structural phase transition across $T_{\mathrm{str}}$. In the antiferromagnetically ordered state below $T_{N}$, both Fe1 and Fe2 magnetic moments lying within the weakly and strongly buckled honeycomb layers are arranged in a fashion that the three nearest neighbors are directed oppositely. Upon cooling below $T_{\text {str }}$, the symmetry of crystal structure is lowered from trigonal $P-3 c 1$ to monoclinic $C 2 / c$, in which a weak sliding of the metal octahedral planes introduces a monoclinic distortion of $\sim 1.7^{\circ}$. The magnetic structure is preserved in the low-temperature monoclinic phase, and the Fe magnetic moment increases from 2.1(1) $\mu_{B}$ at $95 \mathrm{~K}$ to 3.83(4) $\mu_{B}$ at $10 \mathrm{~K}$ assuming an equal moment configuration at $\mathrm{Fe} 1$ and $\mathrm{Fe} 2$ sites. The magnetic point group and linear magnetoelectric tensor at each temperature region are determined. From a symmetry-related tensor analysis, the microscopic origins of the magnetoelectric effects between $T_{N}$ and $T_{\text {str }}$ are proved to be spin-current and $d$ - $p$ hybridization mechanisms.
\end{abstract}

DOI: 10.1103/PhysRevB.100.094109

\section{INTRODUCTION}

Multiferroic materials have been extensively studied over the last two decades since they hold great application possibilities in rapidly growing information technology such as the storage memory or logic devices. In multiferroic materials, ferroelectric and magnetic orders can coexist and have a mutual coupling between them. Therefore, the electric ordering can be controlled by changing the magnetic field and vice versa. Because these two ordering parameters are usually mutually exclusive, multiferroics are not commonly found in nature. Thus, an enormous effort has been devoted to predict and design multiferroic materials with outstanding magnetoelectric (ME) coupling. In this regard, spin-driven (or type-II) multiferroics are promising candidates in which some specific types of magnetic structure break the inversion symmetry and generate polarization. Several type-II multiferroics such as $\mathrm{TbMnO}_{3}, \mathrm{TbMn}_{2} \mathrm{O}_{5}, \mathrm{CuO}$, and $\mathrm{GdFeO}_{3}$ have been found to exhibit strong ME coupling [1-4].

\footnotetext{
*ja.alonso@icmm.csic.es

†yschai@cqu.edu.cn

¥jgcheng@iphy.ac.cn
}

Apart from this class of multiferroics, another group of materials possessing centrosymmetric crystal structure, e.g., $\mathrm{Cr}_{2} \mathrm{O}_{3}, \mathrm{FeSb}_{2} \mathrm{O}_{4}, \mathrm{NdCrTiO}_{5}$, and $\mathrm{MnTiO}_{3}$ [5-8], also exhibit strong cross coupling between electric and magnetic order via the linear magnetoelectric (LME) effect. Unlike type-II multiferroics, they are not ferroelectrics at the ground state, and become ferroelectric only under an external magnetic field above some critical value. In addition, their polarizations linearly increase with magnetic field. Although the LME effect was first observed in antiferromagnetic (AF) $\mathrm{Cr}_{2} \mathrm{O}_{3}$ in 1961 [5], so far only a limited number of LME materials are available due to symmetry restriction.

Recently, the family of $A_{4} \mathrm{M}_{2} \mathrm{O}_{9}(A=\mathrm{Fe}, \mathrm{Co}, \mathrm{Mn}$ and $M=\mathrm{Nb}, \mathrm{Ta})$ materials has attracted profound research interest owing to the observation of novel LME effect [9-17]. At ambient conditions, these materials crystallize in a trigonal $P-3 c 1$ centrosymmetric space group, analogous to the magnetoelectric corundum-type $\mathrm{Cr}_{2} \mathrm{O}_{3}$ [18]. In a more recent study on $\mathrm{Fe}_{4} \mathrm{Nb}_{2} \mathrm{O}_{9}$ (FNO), LME coupling and spin-driven ferroelectricity were reported below its Néel temperature, $T_{N}$, under magnetic field above $6 \mathrm{~T}$ [9]. Its magnetic susceptibility $\chi(T)$ exhibits an anomaly at the paramagnetic to $\mathrm{AF}$ transition of $T_{N} \approx 90 \mathrm{~K}$. Interestingly, its dielectric permittivity $\varepsilon^{\prime}(T)$ 
displays two successive anomalies at about 90 and $77 \mathrm{~K}$, respectively. While the former anomaly corresponds to the $\mathrm{AF}$ transition seen in $\chi(T)$, the origin for the second anomaly of $\varepsilon^{\prime}(T)$ around $77 \mathrm{~K}$ remains elusive. In Ref. [9], it was speculated that the latter anomaly might arise from either a spin reorientation or even a structural transition. To address this issue, it is essential to obtain the information about lowtemperature crystal and magnetic structures of FNO, which remain unknown to our knowledge. In this work, we have carried out low-temperature neutron-powder-diffraction (NPD) measurements on polycrystalline FNO. Detailed data analyses allowed us to determine its AF spin structure below $T_{N}$, and to reveal a structural phase transition across $T_{\text {str }}$ (denoted as $T_{N 2}$ in Ref. [9]) from high-temperature trigonal $P-3 c 1$ to low-temperature monoclinic $C 2 / c$ phase.

\section{EXPERIMENTAL}

Polycrystalline FNO samples were prepared in a conventional solid-state reaction route as described in Ref. [9]. Stoichiometric amounts of $\mathrm{Fe}, \mathrm{Fe}_{2} \mathrm{O}_{3}$, and $\mathrm{Nb}_{2} \mathrm{O}_{5}$ powders were thoroughly mixed and then pelletized in a glove box filled with argon. The pellets were then sealed under vacuum in a quartz tube and heated at $1000^{\circ} \mathrm{C}$ for $20 \mathrm{~h}$. This process was repeated once to improve the sample quality. Powder x-ray diffraction (XRD) was carried out at room temperature to verify the phase purity using $\mathrm{Cu} \mathrm{K}$ radiation. NPD measurements were carried out on the High Resolution Powder Diffractometer at China Advanced Research Reactor, using neutron wavelength $\lambda=1.888 \AA$ in the angular range $7^{\circ}-152^{\circ}$ with a step of $0.07^{\circ}$. The data have been collected at $150,110,95,85$, 60 , and $10 \mathrm{~K}$. Temperature-dependent dielectric permittivity was measured with Agilent E4980A Precision LCR meter at different frequencies. The low-temperature environment was realized in a Physical Property Measurement System (PPMS) from Quantum Design (QD). Magnetic susceptibility was measured with a QD Magnetic Property Measurement System (MPMS3) magnetometer.

\section{RESULTS}

Room-temperature XRD pattern on FNO confirms that we have obtained a nearly single-phase sample with $\mathrm{Nb}_{2} \mathrm{Co}_{4} \mathrm{O}_{9}$ type structure having a trigonal $P-3 c 1$ space group. A small amount of $\mathrm{FeNb}_{2} \mathrm{O}_{6}$ secondary phase, $\sim 1.3 \mathrm{wt} \%$, was identified via analyzing the XRD pattern, similar as reported in the previous study [9]. The calculated lattice parameters $a=$ 5.2231(1) $\AA$ and $c=14.2018(4) \AA$ at room temperature are in excellent agreement with those of $a=5.2253(1) \AA$ and $c=14.2058(2) \AA$ reported previously [9].

The obtained sample is further characterized by measurements of magnetic susceptibility $\chi(T)$ and dielectric permittivity $\varepsilon^{\prime}(T)$. The $\chi(T)$ was measured upon warming up under an external field of $0.01 \mathrm{~T}$ after both zero-field cooled (ZFC) and field cooled (FC) from room temperature. As shown in Fig. 1(a), a small kink observed in both ZFC and FC $\chi(T)$ curves suggests the AF transition around $T_{N} \approx 94 \mathrm{~K}$, which is slightly higher than that reported in Ref. [9]. The increase of $\chi(T)$ below $T_{N}$ upon cooling should arise from the field-induced spin canting along some easy axis, which can

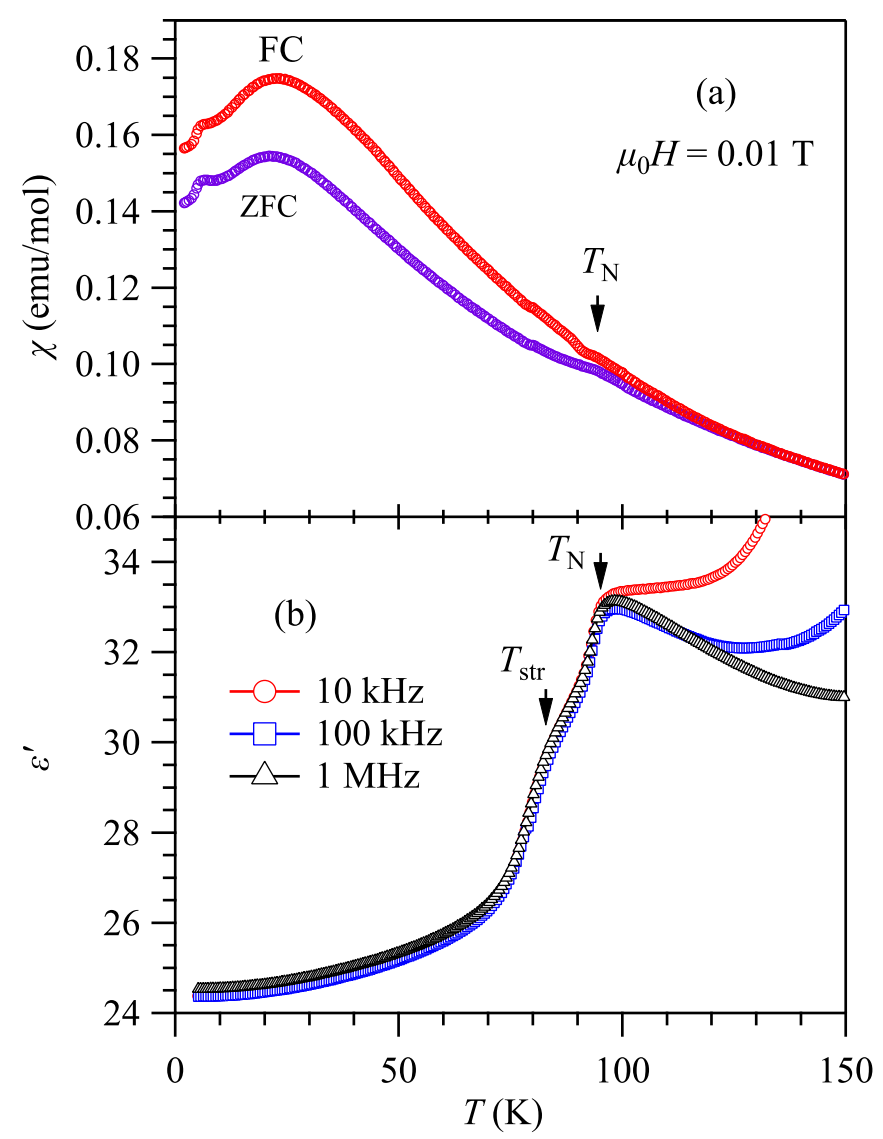

FIG. 1. Temperature dependence of (a) magnetic susceptibility $\chi(T)$ measured under $0.01 \mathrm{~T}$ and $(\mathrm{b})$ dielectric permittivity $\varepsilon^{\prime}(T)$ at different frequencies $(f=10 \mathrm{kHz}, 100 \mathrm{kHz}$, and $1 \mathrm{MHz})$. The Néel temperature $T_{N} \approx 94 \mathrm{~K}$ and the structural transition temperature $T_{\mathrm{str}} \approx 80 \mathrm{~K}$ are indicated by the arrows in the figure.

be determined from measurements of $\chi(T)$ along different directions of single-crystal sample. An additional peak around $6 \mathrm{~K}$ should be attributed to the $\mathrm{FeNb}_{2} \mathrm{O}_{6}$ impurity. Similar as the previous report [9], two anomalies are evidenced in $\varepsilon^{\prime}(T)$ at $T_{N} \approx 94 \mathrm{~K}$ and $T_{\text {str }} \approx 80 \mathrm{~K}$ in the absence of magnetic field. These anomalies show no frequency dependence up to $1 \mathrm{MHz}$ as seen in Fig. 1(b). As mentioned above, the magnetic structure of FNO below $T_{N}$ and the origin of the second $\varepsilon^{\prime}(T)$ anomaly around $T_{\text {str }}$ remain elusive. Thus, the main purpose of this work is to determine the low-temperature crystal and magnetic structures of FNO.

Figure 2 shows the low-angle regions of NPD patterns measured at various temperatures for FNO sample. For the sake of clarity, the patterns are offset by $0.6^{\circ}$ and 600 counts with respect to each other. It can be clearly seen that two consecutive phase transformations take place upon cooling. First, a magnetic transition occurs between 110 and $95 \mathrm{~K}$ as indicated by the emergence of several peaks at low angles around $17^{\circ}, 26^{\circ}$, and $33^{\circ}$. Then, a pronounced peak splitting at $85 \mathrm{~K}$ and below suggests the presence of a structural phase transition between 95 and $85 \mathrm{~K}$. It should be noted that the magnetic and structural transition temperatures seen from NPD in Fig. 2 are slightly higher than those determined from anomalies in $\chi(T)$ and $\varepsilon^{\prime}(T)$ shown in Fig. 1. This is 


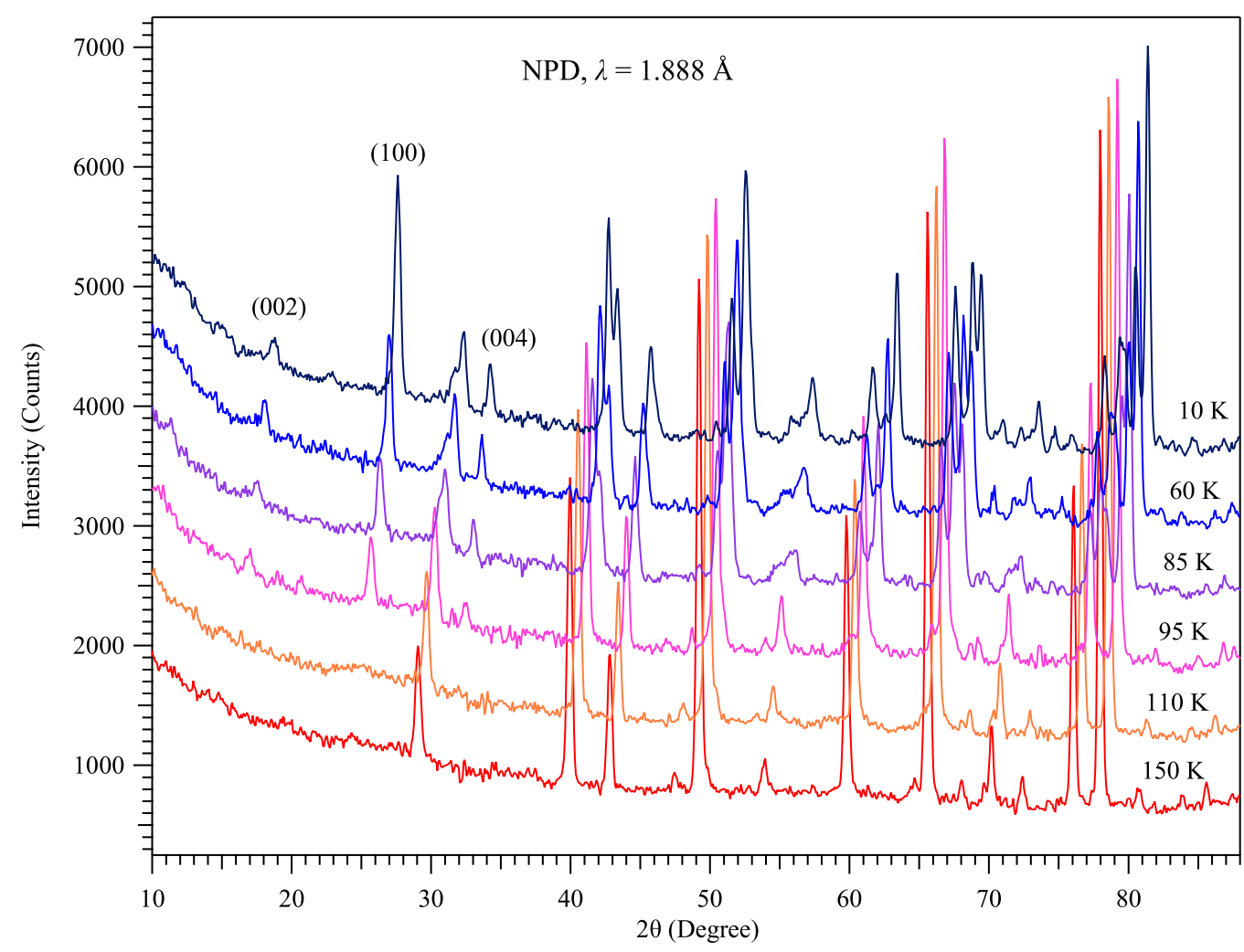

FIG. 2. Neutron-diffraction patterns of $\mathrm{Fe}_{4} \mathrm{Nb}_{2} \mathrm{O}_{9}$ at low-angle regions measured at different temperatures. For the sake of clarity, the patterns are offset by $0.6^{\circ}$ and 600 counts with respect to each other. The $(h k l)$ indices of the magnetic peaks in the figure are given for the high-temperature trigonal structure setting.

presumably due to the distant location of thermometer from sample in the NPD measurements. Thus, the transition temperatures $T_{N} \approx 94 \mathrm{~K}$ and $T_{\text {str }} \approx 80 \mathrm{~K}$ determined from $\chi(T)$ and $\varepsilon^{\prime}(T)$ should be regarded as the real values.

\section{A. Crystal structure at $150 \mathrm{~K}$}

Before solving the low-temperature magnetic and crystal structures, we first determined the structural parameters of FNO at $150 \mathrm{~K}$, which retains the centrosymmetric $P-3 c 1$ symmetry with two $\mathrm{Fe}$ atoms located at $4 d$ sites $(1 / 3,2 / 3, z)$, one $\mathrm{Nb}$ atom at $4 c$ site $(0,0, z)$, and two $\mathrm{O}$ atoms at $6 g$ and $12 f$ positions, respectively. Figure 3(a) displays the Rietveld refinement plot of the NPD data at $150 \mathrm{~K}$. The obtained structural parameters are listed in Table I, and the refined crystal structure is depicted in Fig. 4(a).

As can be seen, the layered trigonal crystal structure represents an ordered stacking of the honeycomb layers of edge-sharing metal-oxygen octahedra, where $(\mathrm{Fe} 2) \mathrm{Nb}$ bilayers are interleaved with a single layer composed of solely Fe1-oxygen octahedra. In this arrangement, every metaloxygen octahedron shares three edges with neighboring metal-oxygen octahedra within the plane, and one face with an octahedron on a neighboring layer along the $c$ axis, while the opposite face borders the vacant space in the middle of the honeycomb hexagons in the neighboring layer of the opposite direction. Along the $c$ axis, Fe1 octahedra are exclusively facing the $\mathrm{Fe} 2$ octahedra and vice versa, and the $\mathrm{Nb}$ octahedra are exclusively facing the $\mathrm{Nb}$ octahedra. In the $(\mathrm{Fe} 2) \mathrm{Nb}$ bilayers, all honeycomb units are exclusively composed of the sequentially positioned $\mathrm{Fe} 2$ and $\mathrm{Nb}$ atoms, such that every $\mathrm{Nb}$ atom borders only the $\mathrm{Fe} 2$ octahedra in the plane, and every $\mathrm{Fe} 2$ atom borders the octahedra around $\mathrm{Nb}$ atoms. The fact that every metal octahedron along the $c$ axis is face-sharing connected to another octahedron in one direction and to the empty space in the middle of a honeycomb unit in another leads to a rather pronounced buckling of the layers. Thereby, the "thickness" of the buckled (Fe2) Nb layers, amounting to $0.715(11) \AA$, is significantly larger than the buckling of the layers composed of solely Fe1 atoms that amounts to $0.420(9)$ $\AA$. The in-plane interatomic separations between the metal atoms in the buckled planes are slightly longer than the separations through the shared faces along the $c$ axis. For example, the $\mathrm{Nb}-\mathrm{Fe} 2$ bonds in the $\mathrm{Fe}(2) \mathrm{Nb}$ planes are 3.098(2) $\AA$, while the $\mathrm{Nb}-\mathrm{Nb}$ bonds along the $c$ axis are 3.081(10) $\AA$, respectively. Similarly, the $\mathrm{Fe} 1-\mathrm{Fe} 1$ bonds in the $\mathrm{Fe} 1$ planes are 3.043(1) $\AA$, while the $\mathrm{Fe} 1-\mathrm{Fe} 2$ bonds along the $c$ axis are 2.931(9) А̊.

\section{B. Magnetic structure at $95 \mathrm{~K}$}

Upon cooling to $95 \mathrm{~K}$, we observe the appearance of (002), (100), (004), and some other diffraction peaks as shown in Fig. 2. All these are actually allowed diffraction peaks for the $P-3 c 1$ crystal structure, yet they have diminishingly weak intensities in neutron diffraction on the purely chemical structure. Thus, they should be attributed to the development of long-range AF ordering with propagation vector $\kappa=0$. By 


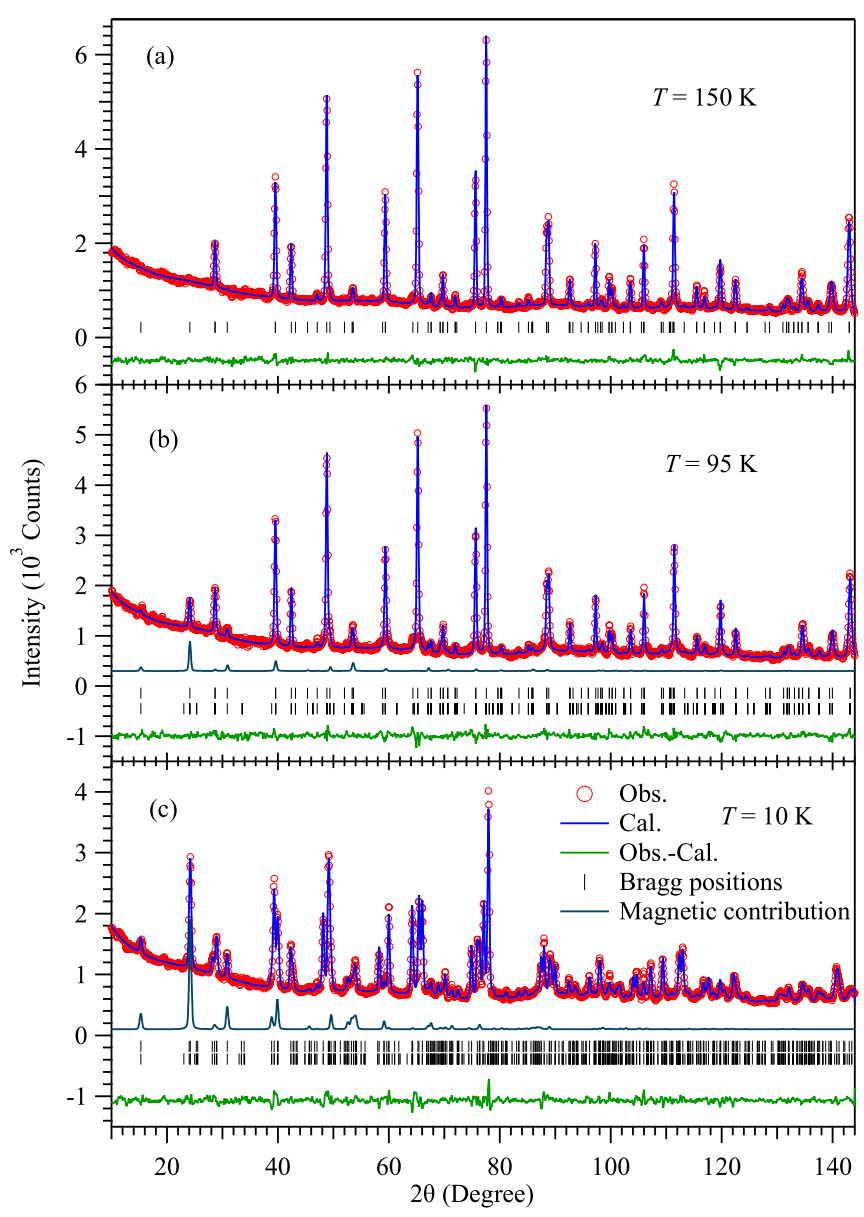

FIG. 3. Rietveld refinement plots of the neutron-powderdiffraction data at (a) 150, (b) 95, and (c) $10 \mathrm{~K}$. The ticks below the graphs indicate the calculated Bragg positions of the crystal structure (upper row) and magnetic structure (lower row). The magnetic contributions at 95 and $10 \mathrm{~K}$ are also shown under the observed/calculated profiles.

using the program SARAH [19], we carried out the symmetry analysis of possible magnetic ordering of the $\mathrm{Fe} 1$ and $\mathrm{Fe} 2$ ions for a propagation vector $\kappa=0$, based on the NPD at $95 \mathrm{~K}$ and high-temperature trigonal crystal structure. Decomposition of the magnetic representation $\Gamma_{\mathrm{Mag}}$ and the possible magnetic basis vectors are summarized in Supplemental

TABLE I. Crystal structural parameters of $\mathrm{Fe}_{4} \mathrm{Nb}_{2} \mathrm{O}_{9}$ refined from neutron-powder-diffraction data at $150 \mathrm{~K}^{\mathrm{a}}$ in space group $P-3 c 1$ (No. $165, Z=2$ ) with the lattice parameters $a=5.2205(2) \AA, c=$ 14.1857(5) $\AA$, and $V=334.81(2) \AA^{3}$.

\begin{tabular}{lccccl}
\hline \hline Atom & Site & $x$ & $y$ & $z$ & $B\left(\AA^{2}\right)$ \\
\hline $\mathrm{Nb}$ & $4 c$ & 0 & 0 & $0.1414(5)$ & $0.61(11)$ \\
$\mathrm{Fe} 1$ & $4 d$ & $1 / 3$ & $2 / 3$ & $0.0148(4)$ & $0.59(13)$ \\
$\mathrm{Fe} 2$ & $4 d$ & $1 / 3$ & $2 / 3$ & $0.3082(5)$ & $0.82(12)$ \\
$\mathrm{O} 1$ & $12 g$ & $0.3403(6)$ & $0.3158(11)$ & $0.0849(4)$ & $0.66(7)$ \\
$\mathrm{O} 2$ & $6 f$ & $0.2824(10)$ & 0 & $1 / 4$ & $0.58(12)$ \\
\hline \hline
\end{tabular}

aReliability factors: $\chi^{2}=1.58, R_{\mathrm{Bragg}}(\%)=5.14$.
Material, Table S1 [20]. After a careful examination of all possible magnetic-ordering models against the NPD pattern in the Rietveld refinements, we conclude that the magnetic ordering occurs according to the irreducible representation $\Gamma_{6}$, and the magnetic structure can be described by a linear combination of either the basis vectors $\psi_{9}$ and $\psi_{10}$, or $\psi_{11}$ and $\psi_{12}$. The coefficients in these linear combinations are identical, i.e., either of the two combinations $1^{*} \psi_{9}+1 / 3^{*} \psi_{10}$ or $1^{*} \psi_{11}+1 / 3^{*} \psi_{12}$, provides an equivalently satisfying fit to the experimental data, and gives an essentially identical magnetic structure.

An illustration of the final Rietveld refinement of the crystal and magnetic structures based on the NPD data at $95 \mathrm{~K}$ is shown in Fig. 3(b). For a better understanding of the magnetic structure, it is instructive to map out the magnetic $\mathrm{Fe}$ sublattices. The two distinct honeycomb $\mathrm{Fe}$ layers are highlighted in Fig. 4(c). The Fe1 atoms form a weakly buckled $\mathrm{Fe} 1$ layer with the interatomic separations of 3.043(1) $\AA$ and an effective buckling thickness of $0.420(9) \AA$, while the $\mathrm{Fe} 2$ atoms belonging to two adjacent $(\mathrm{Fe} 2) \mathrm{Nb}$ layers form a strongly buckled $\mathrm{Fe} 2$ layer, in which both the interatomic $\mathrm{Fe} 2-\mathrm{Fe} 2$ distances of 3.437(5) $\AA$, and the buckling thickness of 1.651(11) $\AA$ are much larger than those in the Fe1 layer. In addition, while the $\mathrm{Fe} 1$ layer is indeed a unique layer formed by the edge-sharing octahedra around the Fe1 atoms, the exact exchange paths in the severely buckled Fe2 layer are more complex, because these $\mathrm{Fe} 2$ atoms are consecutively belonging to different neighboring ( $\mathrm{Fe} 2) \mathrm{Nb}$ layers.

The refined magnetic structure at $95 \mathrm{~K}$ is depicted in Fig. 4(d). As can be seen, the $\mathrm{Fe} 1$ and $\mathrm{Fe} 2$ magnetic moments are lying in the $a b$ plane, and are arranged antiferromagnetically inside the planes. For every Fe atom in either the weakly or strongly buckled honeycomb layers, the moments of the three nearest-neighbor Fe atoms in the same layer are directed oppositely. Along the $c$ axis, the moments of $\mathrm{Fe} 1$ and $\mathrm{Fe} 2$ atoms are coupled ferromagnetically. Assuming an equal moment configuration at the $\mathrm{Fe} 1$ and $\mathrm{Fe} 2$ sites, the magnitude of Fe magnetic moment is refined to be $2.1(1) \mu_{B}$ at $95 \mathrm{~K}$.

\section{Structural phase transition at $85 \mathrm{~K}$}

Upon further cooling to $85 \mathrm{~K}$ and below, the nuclear diffraction peaks get split (Fig. 2), which indicates that the sample undergoes a structural phase transition. A closer inspection of the NPD patterns at $85 \mathrm{~K}$ and below indicates that nuclear-diffraction peaks display a specific splitting pattern, whereas the magnetic peaks seem to stay unchanged in their positions except for a gradual enhancement of intensity upon cooling. It was thus assumed that the structure transition should happen into the symmetry-related subgroup, while the magnetic structure is preserved. Indeed, a direct subgroup of the $P-3 c 1$-a monoclinic $C 2 / c$ space group-immediately delivers a solution that can satisfactorily describe the observed pattern.

In the low-temperature monoclinic $C 2 / c$ structure, the positions of $\mathrm{O} 1$ and $\mathrm{O} 2$ atoms of the high-temperature trigonal structure are splitting into three and two independent positions, respectively. Except for O2_1 located at the $4 e$ $(0, y, 1 / 4)$ site, all other atoms are located in the general $8 f(x, y, z)$ positions. Refinement of the crystal-structure 


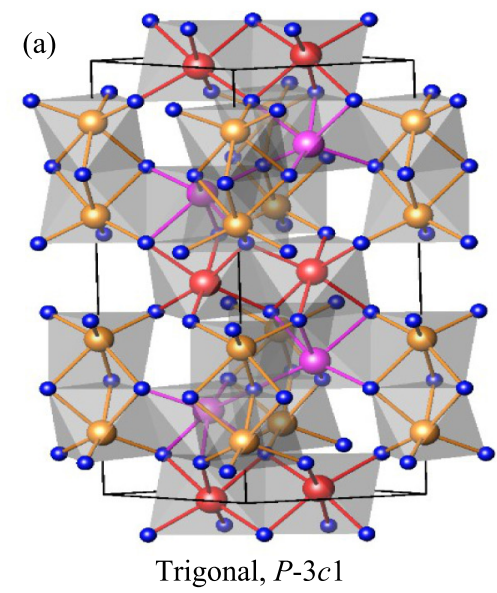

(c)

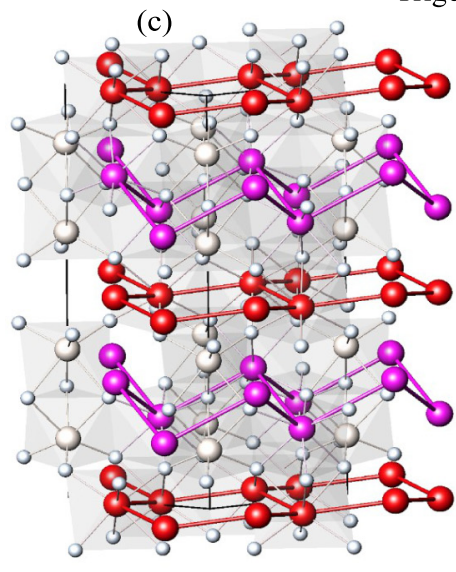

(d)

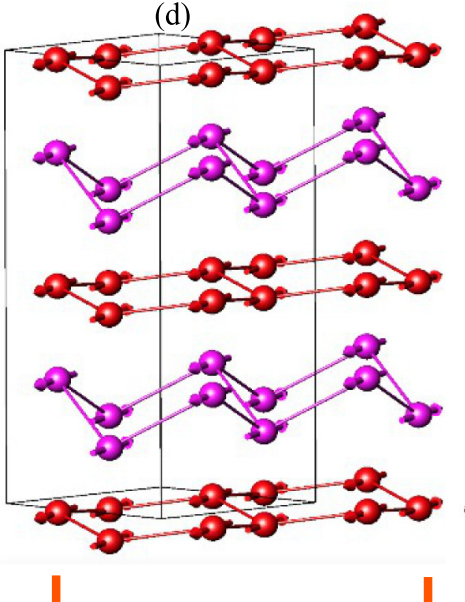

(b)

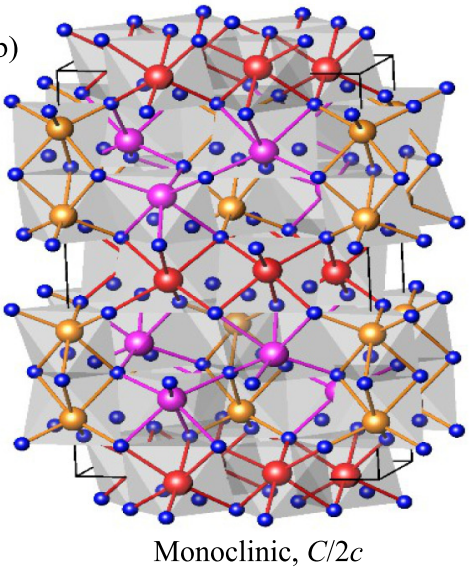

$T_{\mathrm{N}} \approx 95 \mathrm{~K}$

$T_{\text {str }} \approx 85 \mathrm{~K}$

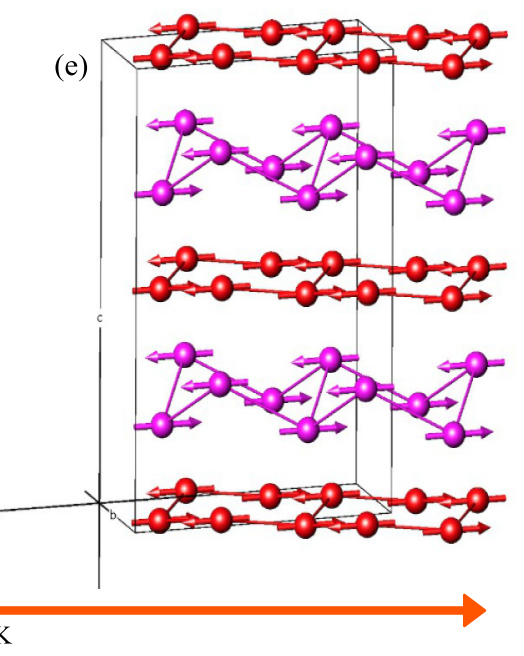

FIG. 4. Illustrations of the crystal structure (a) at $150 \mathrm{~K}$ in trigonal $P-3 c 1$ space group and (b) at $10 \mathrm{~K}$ in monoclinic $C / 2 c$ space group. Fe atoms are plotted in red ( $\mathrm{Fe}$ 1, in the layers formed solely by $\mathrm{Fe}$ ) and in pink ( $\mathrm{Fe} 2$, in the $\mathrm{FeNb}$ layers), $\mathrm{Nb}$ atoms in orange, and oxygen atoms in blue. (c) Schematic view of Fe sublattices in a direction nearly perpendicular to the $c$ axis. The weakly buckled honeycomb layers of Fe1 atoms (red) are interleaved by the strongly buckled honeycomb layers of Fe2 atoms (pink). (d) Schematic view onto the magnetic structure at $95 \mathrm{~K}$ in trigonal phase. Fe moments are directed along the projections of two of the bonds of every honeycomb onto the $x-y$ plane, and $60^{\circ}$ inclined to the projections of the other four bonds. (e) The magnetic structure at $10 \mathrm{~K}$ in the monoclinic phase.

parameters was done based on the neutron data at $10 \mathrm{~K}$. An illustration of the Rietveld refinement plot is shown in Fig. 3(c), and a schematic representation of the crystal structure at $10 \mathrm{~K}$ is given in Fig. 4(b). The refined crystal-structure parameters are summarized in Table II. As shown in Fig. 4(b), while the spatial ordering of the $\mathrm{Fe}$ and $\mathrm{Nb}$ octahedra remains essentially same as the trigonal phase, the low-temperature crystal structure features some differences. The $a / b$ ratio of the resulting rectangle built on the $a$ and $b$ lattice constants displays a weak deviation from $\sqrt{3}$, namely $a / \sqrt{3} b \approx 1.013$. Additionally, a rather weak sliding of the metal octahedral planes with respect to each other introduces a monoclinic distortion of $\sim 1.7^{\circ}$.

For this low-temperature monoclinic cell, all magnetic peaks fit nicely into the $\kappa=0$ assumption, thus confirming that the magnetic ordering is preserved through the structural phase transition. Based on the NPD data at $10 \mathrm{~K}$, the symmetry analysis of possible magnetic ordering of the Fe1 and $\mathrm{Fe} 2$ ions, both located in the $8 f(x, y, z)$ positions for a propagation vector $\kappa=0$, has been carried out again using the program SARAH [19]. The decomposition of the magnetic representation $\Gamma_{\mathrm{Mag}}$ and the possible magnetic basis vectors are summarized in Table S2 [20]. After checking all possibilities, we conclude that the only irreducible representation that succeeds to fit the data is the $\Gamma_{2}$ with the $\psi_{4}$ basis function. The resulting magnetic structure is presented in Fig. 4(e). The in-plane contradiction between the trigonal symmetry and the unique direction of magnetic moments along one of the three $\mathrm{Fe}-\mathrm{Fe}$ bonds' directions at $95 \mathrm{~K}$ is being taken off by a slight deviation from trigonal symmetry in the $x y$ plane. Under assumption of the equal moment configuration, the magnetic Fe moments amount to $3.83(4) \mu_{B}$ at $10 \mathrm{~K}$.

\section{DISCUSSION}

The magnetic structure of $A_{4} M_{2} \mathrm{O}_{9}(A=\mathrm{Co}, \mathrm{Mn}$ and $M=$ $\mathrm{Nb}, \mathrm{Ta})$ materials and the origin of spin-induced polarization have been subjected to extensive study. Bertaut et al. [18] first reported the magnetic structure of $\mathrm{Mn}_{4} \mathrm{Nb}_{2} \mathrm{O}_{9}$ and $\mathrm{Co}_{4} \mathrm{Nb}_{2} \mathrm{O}_{9}$ using NPD experiments. According to their study, the spins 
TABLE II. Crystal structural parameters of $\mathrm{Fe}_{4} \mathrm{Nb}_{2} \mathrm{O}_{9}$ refined from neutron-powder-diffraction data at $10 \mathrm{~K}^{\mathrm{a}}$ in space group $C 2 / c$ (No. $15, Z=4$ ) with the lattice parameters $a=$ 9.0916(6) $\mathrm{A}, b=5.1825(3) \AA, c=14.1983(9) \AA, \quad \beta=91.677(1)^{\circ}$, and $V=668.71(7) \AA^{3}$.

\begin{tabular}{lccccc}
\hline \hline Atom & Site & $x$ & $y$ & $z$ & $B\left(\AA^{2}\right)$ \\
\hline $\mathrm{Nb}$ & $8 f$ & $-0.0007(11)$ & $-0.000(3)$ & $0.1421(8)$ & $0.31(18)$ \\
$\mathrm{Fe} 1$ & $8 f$ & $0.1645(8)$ & $0.501(3)$ & $0.0132(5)$ & $0.65(13)$ \\
$\mathrm{Fe} 2$ & $8 f$ & $0.1722(9)$ & $0.505(2)$ & $0.3066(6)$ & $0.97(16)$ \\
$\mathrm{O} 1 \_1$ & $8 f$ & $0.1698(16)$ & $0.850(3)$ & $0.084(1)$ & $0.77(12)^{\mathrm{b}}$ \\
$\mathrm{O} 1 \_2$ & $8 f$ & $0.3462(16)$ & $0.3192(19)$ & $0.087(1)$ & $0.77(12)^{\mathrm{b}}$ \\
$\mathrm{O} 1 \_3$ & $8 f$ & $0.4951(17)$ & $0.825(2)$ & $0.085(1)$ & $0.77(12)^{\mathrm{b}}$ \\
$\mathrm{O} 2 \_1$ & $4 \mathrm{e}$ & 0 & $0.720(4)$ & $1 / 4$ & $1.1(2)^{\mathrm{c}}$ \\
$\mathrm{O} 2 \_2$ & $8 f$ & $0.1365(12)$ & $0.145(3)$ & $0.250(1)$ & $1.1(2)^{\mathrm{c}}$ \\
\hline \hline
\end{tabular}

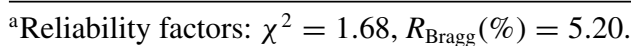

b Atomic displacement parameters for atoms O1_1, O1_2, and O1_3 were refined with constraint to equality.

${ }^{\mathrm{c}}$ Atomic displacement parameters for atoms O2_1 and O2_1 were refined with constraint to equality.

are ordered along the $c$ axis within two ferromagnetic chains along $(1 / 3,2 / 3, z)(+$ spin $)$ and $(2 / 3,1 / 3, z)(-$ spin $)$. These two chains are antiferromagnetically coupled resulting in a net zero magnetization. Magnetic susceptibility measurements on $\mathrm{Mn}_{4} \mathrm{Nb}_{2} \mathrm{O}_{9}$ single crystal confirmed the collinear magnetic structure along the $c$ axis in this system [21]. Similar magnetic structure was also reported in $\mathrm{Mn}_{4} \mathrm{Ta}_{2} \mathrm{O}_{9}$ single crystal [22]. In contrast, measurements of single-crystal neutron diffraction and magnetic susceptibility along different crystallographic directions have shown that the magnetic moments in $\mathrm{Co}_{4} \mathrm{Nb}_{2} \mathrm{O}_{9}$ are confined in the trigonal basal plane instead of parallel to the $c$ axis [12]. Later on, Deng et al. showed that the magnetic moments of Co atoms are noncollinearly arranged in the $a b$ plane [23]. A recent neutron-diffraction study on $\mathrm{Co}_{4} \mathrm{Ta}_{2} \mathrm{O}_{9}$ also evidenced noncollinearly canted spin arrangement of Co atoms in the $a b$ plane [24].

In the present study, we not only determined the magnetic structure of FNO below $T_{N}$, but also uncovered a structural phase transition at $T_{\text {str }}<T_{N}$. It is noteworthy that such a temperature-driven structural phase transition observed in FNO has not been observed in related $A_{4} M_{2} \mathrm{O}_{9}$ ( $A=\mathrm{Mn}, \mathrm{Co})$ materials. The detailed structural parameters of FNO at different temperatures are given in Table S3 [20]. Such important information from this study enriches the magnetic and structural properties of the $A_{4} M_{2} \mathrm{O}_{9}$ family. In contrast to the above-mentioned $A_{4} M_{2} \mathrm{O}_{9}$ materials, the magnetic moments of both $\mathrm{Fe} 1$ and $\mathrm{Fe} 2$ atoms are found to be confined roughly in the $a b$ plane and directed antiparallel with respect to its three nearest neighbors. Despite the distinct magnetic structures, the observation of dielectric anomaly around $T_{N}$ in FNO suggests a strong ME coupling. The magnetic structure determined in this work can provide the basis for further studies of LME coupling in FNO.
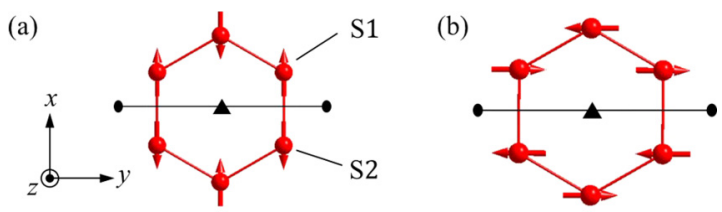

FIG. 5. Top view onto the two possible magnetic structures at $95 \mathrm{~K}$ in trigonal phase. Fe moments are directed along the (a) $x$ axis and (b) $y$ axis.

As mentioned above, our results reveal that $\mathrm{Fe} 1$ and $\mathrm{Fe} 2$ magnetic moments are collinearly arranged in the $a b$ plane with two possibilities. We first define Cartesian coordinates, $x$, $y, z$, as shown in Fig. 5. For $T_{\text {str }}<T<T_{N}$, (1) if the magnetic structure is given by the basis vectors $1^{*} \psi_{9}+1 / 3^{*} \psi_{10}$, the magnetic moments of the $\mathrm{Fe} 1$ and $\mathrm{Fe} 2$ atoms are antiparallelly aligned along the $x / /[2,1,0]$ direction, respectively, as shown in Fig. 5(a). Accordingly, the magnetic point group is $\mathbf{2} / \boldsymbol{m}^{\prime}$. 2 is twofold rotation along the $y / /[0,1,0]$ direction. (2) If the magnetic structure is given by the basis vectors $1^{*} \psi_{11}+1 / 3^{*} \psi_{12}$, the magnetic moments of the $\mathrm{Fe} 1$ and $\mathrm{Fe} 2$ are antiparallelly aligned along the $y$ direction, respectively, as shown in Fig. 5(b). Accordingly, the magnetic point group is $\mathbf{2}^{\prime} / \boldsymbol{m}$. Below $85 \mathrm{~K}$, the magnetic point group is $\mathbf{2} / \boldsymbol{m}^{\prime}$ too. Both $\mathbf{2}^{\prime} / \boldsymbol{m}$ and $\mathbf{2} / \boldsymbol{m}^{\prime}$ are nonpolar and allow LME effect [25]. In particular, the linear magnetoelectric matrix form is

$$
\left[\begin{array}{ccc}
0 & \alpha_{x y} & 0 \\
\alpha_{y x} & 0 & \alpha_{y z} \\
0 & \alpha_{z y} & 0
\end{array}\right]
$$

for $\mathbf{2}^{\prime} / \mathbf{m}$ and

$$
\left[\begin{array}{ccc}
\alpha_{x x} & 0 & \alpha_{x z} \\
0 & \alpha_{y y} & 0 \\
\alpha_{z x} & 0 & \alpha_{z z}
\end{array}\right]
$$

for $\mathbf{2} / \boldsymbol{m}^{\prime}$, respectively, which are consistent with the reported LME behavior below $95 \mathrm{~K}$ [9].

In type-II multiferroic, the ME coupling below the magnetic-ordering temperature has been explained in terms of three well-known models, i.e., the spin-current mechanism for noncollinear magnetic structure [26], the magnetostriction model for collinear spin arrangements [27], and $d$ - $p$ hybridization mechanism between spin and its ligand atoms with spin-orbital interaction [28], in some low-symmetry crystals with noncollinear magnetic orders. In order to extract more information about the microscopic origin of ME coupling, we introduce a symmetry-based local ME tensor technique [29], which has successfully resolved the microscopic origin of polarization in multiferroic hexaferrites [30]. Let us consider that a spin pair $S_{1}$ and $S_{2}$ can produce a local dipole $p$ as the quadratic functions in Einstein convention:

$$
\begin{aligned}
p & =p\left(S_{1}, S_{2}\right)+p\left(S_{1}\right)+p\left(S_{1}\right) \\
& =P_{12}^{\alpha \beta \gamma} S_{1 \alpha} S_{2 \beta}+\sum_{i=1,2} P_{i i}^{\alpha \beta \gamma} S_{i \alpha} S_{i \beta},
\end{aligned}
$$


where $\alpha, \beta, \gamma$ run over all the Cartesian coordinates, $x, y, z$. All three well-known mechanisms mentioned above can be expressed as a special form of spin tensor $P_{i i}^{\alpha \beta \gamma}$ and two-spin tensor $P_{i j}^{\alpha \beta \gamma}$. In particular, the spin-current mechanism $p \propto$ $e_{12} \times\left(S_{1} \times S_{2}\right)$ leads to antisymmetric $P_{i j}^{\alpha \beta \gamma}$ :

$$
\left(\begin{array}{ccc}
0 & e_{12 y},-e_{12 x}, 0 & e_{12 z}, 0,-e_{12 x} \\
-e_{12 y}, e_{12 x}, 0 & 0 & 0, e_{12 z},-e_{12 y} \\
-e_{12 z}, 0, e_{12 x} & 0,-e_{12 z}, e_{12 y} & 0
\end{array}\right)
$$

while exchange-striction mechanism $p \propto e_{12}\left(S_{1} \bullet S_{2}\right)$ shows a $P_{i j}^{\alpha \beta \gamma}$ with diagonal term only:

$$
\left(\begin{array}{ccc}
e_{12 x}, e_{12 y}, e_{12 z} & 0 & 0 \\
0 & e_{12 x}, e_{12 y}, e_{12 z} & 0 \\
0 & 0 & e_{12 x}, e_{12 y}, e_{12 z}
\end{array}\right)
$$

where $e_{12}=\left(e_{12 x}, e_{12 y}, e_{12 z}\right)$ is connecting vector of adjacent spin pair $S_{1}$ and $S_{2}$.

In contrast, $p$ - $d$ hybridization, $p \propto e_{i}\left(e_{i} \bullet S_{i}\right)^{2}$, has a $P_{i i}^{\alpha \beta \gamma}$ tensor with the symmetric matrix form:

$$
\left(\begin{array}{ccc}
e_{i x} e_{i x} e_{i x}, e_{i x} e_{i x} e_{i y}, e_{i x} e_{i x} e_{i z} & e_{i x} e_{i y} e_{i x}, e_{i x} e_{i y} e_{i y}, e_{i x} e_{i y} e_{i z} & e_{i x} e_{i z} e_{i x}, e_{i x} e_{i z} e_{i y}, e_{i x} e_{i z} e_{i z} \\
e_{i y} e_{i x} e_{i x}, e_{i y} e_{i x} e_{i y}, e_{i y} e_{i x} e_{i z} & e_{i y} e_{i y} e_{i x}, e_{i y} e_{i y} e_{i y}, e_{i y} e_{i y} e_{i z} & e_{i y} e_{i z} e_{i x}, e_{i y} e_{i z} e_{i y}, e_{i y} e_{i z} e_{i z} \\
e_{i z} e_{i x} e_{i x}, e_{i z} e_{i x} e_{i y}, e_{i z} e_{i x} e_{i z} & e_{i z} e_{i y} e_{i x}, e_{i z} e_{i y} e_{i y}, e_{i z} e_{i y} e_{i z} & e_{i z} e_{i z} e_{i x}, e_{i z} e_{i z} e_{i y}, e_{i z} e_{i z} e_{i z}
\end{array}\right)
$$

where vector $e_{i}=\left(e_{i x}, e_{i y}, e_{i z}\right)$ connects the transition metal $i$ and its neighbor ligand atom. By introducing the crystal symmetry, the forms of the local ME tensors can be greatly simplified due to its polar tensor property [30]. For simplicity, we assume: (i) there are only three kinds of two-spin interactions that can generate local dipole, Fe1-Fe1, Fe2-Fe2, and $\mathrm{Fe} 1-\mathrm{Fe} 2$. As shown in Fig. 4(d), the Fe1 and Fe2 atoms are denoted as the red and purple symbols, respectively, and the $\mathrm{Fe} 1-\mathrm{Fe} 1$ and $\mathrm{Fe} 2-\mathrm{Fe} 2$ are bonds between the nearest-neighbor Fe atoms within the red and purple layers, having bond length of 3.043 and $3.412 \AA$, respectively. The Fe1-Fe2 bonds are along the $c$ axis between two nearest-neighbor Fe1 and Fe2 atoms with a distance of $2.931 \AA$. All three kinds of bonds between nearest-neighbor $\mathrm{Fe}$ atoms are considered for the local electric dipole; (ii) in any spin configuration, the spins in $\mathrm{Fe} 1$ and $\mathrm{Fe} 2$ layers are identical and respond in same manner to the external field $H$. As shown in Fig. 5, the spin at 1 site in Fe1 layer is $S_{1}$ and 2 site $S_{2}$ where $S_{1}=-S_{2}$ only hold for $H=0$. Then, the total polarization generated in Fe1 layer $p_{\mathrm{Fe} 1-\mathrm{Fe} 1}$ by $\mathrm{Fe} 1-\mathrm{Fe} 1$ two-spin interaction can be expressed as

$$
p_{\mathrm{Fe} 1-\mathrm{Fe} 1}=\sum_{i j} P_{i j}^{\alpha \beta \gamma} S_{i \alpha} S_{j \beta}=\left(\sum_{i j} P_{i j}^{\alpha \beta \gamma}\right) S_{1 \alpha} S_{2 \beta},
$$

where $i j$ runs all adjacent $\mathrm{Fe} 1-\mathrm{Fe} 1$ pairs and ensure that $S_{i}$ is $S_{1}$ and $S_{j}$ is $S_{2}$. After considering all the $\mathbf{3}_{z}, \boldsymbol{2}_{y}$, and $\mathbf{- 1}$ symmetric operations, $\sum_{i j} P_{i j}^{\alpha \beta \gamma}$ can be simplified as

$$
\sum_{i j} P_{i j}^{\alpha \beta \gamma} \propto\left(\begin{array}{ccc}
0 & 0 & f, 0,0 \\
0 & 0 & 0, f, 0 \\
-f, 0,0 & 0,-f, 0 & 0
\end{array}\right) .
$$

For the $\mathrm{Fe} 2-\mathrm{Fe} 2$ and $\mathrm{Fe} 1-\mathrm{Fe} 2$ case, we derived the exactly same form in $\sum_{i j} P_{i j}^{\alpha \beta \gamma}$. Then Eq. (7) can only be expressed in a form similar to the spin-current mechanism:

$$
p_{\mathrm{Fe} 1-\mathrm{Fe} 1} \propto(0,0, f) \times\left(S_{1} \times S_{2}\right),
$$

where $(0,0, f)$ is a vector along $z$. For $H=0, H / / x$, and $H / / y,\left(S_{1} \times S_{2}\right)=0$ or $/ / z$ so that $p_{F e 1-F e 1}=0$. For $H / / z$, the $\left(S_{1} \times S_{2}\right) / / y$ and $/ / x$ for $\mathbf{2} / \boldsymbol{m}^{\prime}$ and $\mathbf{2}^{\prime} / \boldsymbol{m}$ point group, respectively. Then, $p_{F e 1-F e 1}$ should be along $x$ and $y$ direction, respectively, corresponding to nonzero $\alpha_{x z}$ and $\alpha_{y z}$ ME coefficients in $\mathbf{2} / \boldsymbol{m}^{\prime}$, and $\mathbf{2}^{\prime} / \boldsymbol{m}$, respectively, in Eqs. (1) and (2). The same argument could be applicable to Fe2-Fe2 interactions. However, for Fe2-Fe1, the two spins are the same due to the ferromagnetic along the $c$ axis, leading to zero net polarization. Therefore, we can draw the conclusion that only spin-current mechanism can contribute to $\alpha_{x z}$ or $\alpha_{y z}$ by Fe1-Fe1 and Fe2-Fe2 interactions. Exchange-striction and anisotropic-symmetric-exchange mechanisms [31] due to spin-orbital coupling can be completely ruled out in the linear ME effect from symmetry analysis [Eq. (9)].

As for the single-spin induced polarization $p_{\mathrm{Fe}}$, it can be expressed as

$$
p_{\mathrm{Fe}}=\sum_{i} P_{i i}^{\alpha \beta \gamma} S_{1 \alpha} S_{1 \beta}+\sum_{j} P_{j j}^{\alpha \beta \gamma} S_{2 \alpha} S_{2 \beta},
$$

where $S_{1}$ spin resides on the $i$ sites and $S_{2}$ spin on the $j$ sites. After considering all the $\mathbf{3}_{z}, \mathbf{2}_{y}$, and $\mathbf{- 1}$ symmetric operations, $\sum_{i} P_{i i}^{\alpha \beta \gamma}$ and $\sum_{j} P_{j j}^{\alpha \beta \gamma}$ can be simplified as

$$
\begin{gathered}
\sum_{i} P_{i i}^{\alpha \beta \gamma}=A\left(\begin{array}{ccc}
g, 0, h & 0,-g, 0 & k, 0,0 \\
0,-g, 0 & -g, 0, h & 0, k, 0 \\
k, 0,0 & 0, k, 0 & 0,0, l
\end{array}\right), \\
\sum_{j} P_{j j}^{\alpha \beta \gamma}=-A\left(\begin{array}{ccc}
g, 0, h & 0,-g, 0 & k, 0,0 \\
0,-g, 0 & -g, 0, h & 0, k, 0 \\
k, 0,0 & 0, k, 0 & 0,0, l
\end{array}\right),
\end{gathered}
$$

where $g, h, k, l$, and $A$ are constants. Comparing to Eq. (6) of the $d$ - $p$ hybridization mechanism, $g, h, k, l$ can be nonzero. One could immediately find that $p_{\mathrm{Fe}}=0$ at $H=0$. After applying $H / / x, y$, or $z$, the electric dipole from two spin sites will no longer cancel each other out due to $S_{1}+S_{2} \neq 0$, leading to all allowed nonzero ME coefficients in Eqs. (1) and (2). Therefore, the spin-current mechanism is responsible for one of the ME coefficients, while the $d$-p hybridization mechanism can be the origin of every ME coefficient allowed in Eqs. (1) and (2) between $T_{\text {str }}$ and $T_{N}$. Due to the lower crystal symmetry at $T<T_{\text {str }}$, the mathematical expressions for two-spin and single-spin ME tensor become very complex 
and cannot be simplified further. It is possible that all three main mechanisms are the microscopic origins of linear $\mathrm{ME}$ effects in FNO.

In conclusion, we have investigated the crystal and magnetic structures of the recently discovered magnetoelectric materials $\mathrm{Fe}_{4} \mathrm{Nb}_{2} \mathrm{O}_{9}$ by means of neutron-powder diffraction at low temperatures. We have successfully established the antiferromagnetic spin structure below $T_{N} \approx 94 \mathrm{~K}$, which is featured by antiparallel alignment of Fe moments with respect to its three nearest neighbors within the buckled honeycomb layers. Our results also reveal a structural transition from trigonal $P-3 c 1$ to monoclinic $C 2 / c$ upon cooling across $T_{\text {str }} \approx$ $80 \mathrm{~K}$, which should be responsible for the observed second dielectric anomaly. The magnetic structure is found to be preserved in the low-temperature monoclinic phase, and the Fe magnetic moment increases from $2.1(1) \mu_{B}$ at $95 \mathrm{~K}$ to $3.83(4) \mu_{B}$ at $10 \mathrm{~K}$ assuming an equal moment configuration. We have determined the magnetic point group and linear magnetoelectric tensor at each temperature region, and proved that the microscopic origins of the ME effects between $T_{N}$ and $T_{\text {str }}$ are the spin-current and $d$ - $p$ hybridization mechanisms based on a symmetry-related tensor analysis.

\section{ACKNOWLEDGMENTS}

This work is supported by the National Key R\&D Program of China (Grant No. 2018YFA0305700), the National Natural Science Foundation of China (Grants No. 11574377, No. 11834016, No. 11874400, No. 51772323, and No. 11674384), the Beijing Natural Science Foundation (Grant No. Z190008), the Strategic Priority Research Program and Key Research Program of Frontier Sciences of the Chinese Academy of Sciences (Grants No. XDB25000000 and No. QYZDB-SSWSLH013), and the CAS Interdisciplinary Innovation Team. J.A.A. gratefully acknowledges the Spanish MINECO for granting Project No. MAT2017-84496-R.
[1] T. Kimura, T. Goto, H. Shintani, K. Ishizaka, T. Arima, and Y. Tokura, Nature (London) 426, 55 (2003).

[2] N. Hur, S. Park, P. A. Sharma, J. S. Ahn, S. Guha, and S.-W. Cheong, Nature (London) 429, 392 (2004).

[3] T. Kimura, Y. Sekio, H. Nakamura, T. Siegrist, and A. P. Ramirez, Nat. Mater. 7, 291 (2008).

[4] Y. Tokunaga, N. Furukawa, H. Sakai, Y. Taguchi, T. Arima, and Y. Tokura, Nat. Mater. 8, 558 (2009).

[5] D. N. Astrov, Zh. Eksp. Teor. Fiz. (U.S.S.R.) 40, 1035 (1961) [Sov. Phys. JETP 13, 729 (1961)].

[6] A. Iyama, Y. Wakabayashi, N. Hanasaki, and T. Kimura, Jpn. J. Appl. Phys. 53, 05FB02 (2014).

[7] J. Hwang, E. S. Choi, H. D. Zhou, J. Lu, and P. Schlottmann, Phys. Rev. B 85, 024415 (2012).

[8] N. Mufti, G. R. Blake, M. Mostovoy, S. Riyadi, A. A. Nugroho, and T. T. M. Palstra, Phys. Rev. B 83, 104416 (2011).

[9] A. Maignan and C. Martin, Phys. Rev. B 97, 161106(R) (2018).

[10] S. N. Panja, L. Harnagea, J. Kumar, P. K. Mukharjee, R. Nath, A. K. Nigam, and S. Nair, Phys. Rev. B 98, 024410 (2018).

[11] Y. Fang, Y. Q. Song, W. P. Zhou, R. Zhao, R. J. Tang, H. Yang, L. Y. Lv, S. G. Yang, D. H. Wang, and Y. W. Du, Sci. Rep. 4, 3860 (2014).

[12] N. D. Khanh, N. Abe, H. Sagayama, A. Nakao, T. Hanashima, R. Kiyanagi, Y. Tokunaga, and T. Arima, Phys. Rev. B 93, 075117 (2016).

[13] Y. P. Lu, C. X. Ji, Y. L. Sun, Y. Fang, L. Zhang, Z. D. Han, B. Qian, X. F. Jiang, and W. P. Zhou, J. Alloys Compd. 679, 213 (2016).

[14] Y. M. Xie, C. S. Lin, H. Zhang, and W. D. Cheng, AIP Adv. 6, 045006 (2016).

[15] A. Maignan and C. Martin, Phys. Rev. Mater. 2, 091401(R) (2018).
[16] N. D. Khanh, N. Abe, S. Kimura, Y. Tokunaga, and T. Arima, Phys. Rev. B 96, 094434 (2017).

[17] B. B. Liu, Y. Fang, Z. D. Han, S. M. Yan, W. P. Zhou, B. Qian, D. H. Wang, and Y. W. Du, Mater. Lett. 164, 425 (2016).

[18] E. F. Bertaut, L. Corliss, F. Forrat, R. Aleonard, and R. Pauthenet, J. Phys. Chem. Solids 21, 234 (1961).

[19] A. S. Wills, Physica B 276-278, 680 (2000).

[20] See Supplemental Material at http://link.aps.org/supplemental/ 10.1103/PhysRevB.100.094109 for the decomposition of the magnetic representation and the possible magnetic basis vectors for determining the magnetic structure at 95 and $10 \mathrm{~K}$, as well as the refined structural parameters at different temperatures.

[21] Y. Cao, M. Xiang, Z. Feng, B. Kang, J. Zhang, N. Guiblin, W. Ren, B. Dkhil, and S. Cao, RSC Adv. 7, 13846 (2017).

[22] Y. Cao, K. Xu, Y. Yang, W. Yang, Y. Zhang, Y. Kang, X. He, A. Zheng, M. Liu, S. Wei, Z. Li, and S Cao, J. Cryst. Growth 492, 35 (2018).

[23] G. Deng, Y. Cao, W. Ren, S. Cao, A. J. Studer, N. Gauthier, M. Kenzelmann, G. Davidson, K. C. Rule, J. S. Gardner, P. Imperia, C. Ulrich, and G. J. McIntyre, Phys. Rev. B 97, 085154 (2018).

[24] S. Chaudhary, P. Srivastava, S. D. Kaushik, V. Siruguri, and S. Patnaik, J. Magn. Magn. Mater. 475, 508 (2019).

[25] R. E. Newnham, Properties of Materials: Anisotropy, Symmetry, Structure (Oxford University Press, New York, USA, 2005).

[26] H. Katsura, N. Nagaosa, and A. V. Balatsky, Phys. Rev. Lett. 95, 057205 (2005).

[27] S. Greenwald and J. S. Smart, Nature (London) 166, 523 (1950).

[28] T.-H. Arima, J. Phys. Soc. Jpn. 76, 073702 (2007).

[29] H. J. Xiang, E. J. Kan, Y. Zhang, M.-H. Whangbo, and X. G. Gong, Phys. Rev. Lett. 107, 157202 (2011).

[30] Y. S. Chai, S. H. Chun, J. Z. Cong, and K. H. Kim, Phys. Rev. B 98, 104416 (2018).

[31] J. S. Feng and H. J. Xiang, Phys. Rev. B 93, 174416 (2016). 